\title{
BCGiosis as a presenting feature of a child with chronic granulomatous disease
}

\begin{abstract}
Bacillus Calmette Guerin (BCG) vaccine, which is administered to all newborns in some regions, could lead to serious complication ranging from local disease (known as BCGitis) to disseminated disease (BCGosis) in a group of patients with primary immunodeficiency diseases. We are reporting here a 3.5 year-old girl with a history of prolonged BCGitis, which developed to disseminated disease without any other special features. Immunological studies with nitro-blue tetrazolium test confirmed the diagnosis of chronic granulomatous disease in this patient. Chronic granulomatous disease should be considered in the list of differential diagnosis in all children with BCGosis, even in the absence of any other manifestations related to immunodeficiency.
\end{abstract}

Keywords: BCG vaccine; Mycobacterium bovis; granulomatous disease; chronic.

[Braz J Infect Dis 2011;15(1)83-86]@Elsevier Editora Ltda.

\section{INTRODUCTION}

Chronic granulomatous disease (CGD) is a congenital hereditary disorder of the immune system, characterized by absence of superoxide and hydrogen peroxide production in phagocytes. ${ }^{1-3}$ CGD is inherited as an $\mathrm{x}$-linked recessive trait or as an autosomal recessive. ${ }^{3,4}$

The patients with CGD may present variable manifestations, but severe infections in infancy are the most common presenting features. ${ }^{5-7}$ As the disease affects macrophages and neutrophils, CGD leads to recurrent symptomatic cutaneous, subcutaneous and bone infections as well as abscess formation and lymphadenitis. Lower respiratory tract infections, especially caused by microorganisms that form abscesses and pneumatoceles, are also common in the course of disease. ${ }^{7,8}$

Here, a unique feature of the disease is described in a child primarily infected with Bacillus Calmette Guerin (disseminated BCG) after routine vaccination for BCG in a national vaccination program.

\section{CASE REPORT}

A 3.5 year-old girl was referred to the Children's Medical Center in Tehran, Iran with complaint of an overgrowing axillary mass protruding to the anterior chest wall. The mass was primarily detected as a nodule in early life; as the parents could recall, it originated at the time of early vaccination at neonatal period. However, the nodule has recently begun to grow in size throughout the last four months. The parents were also apprehensive due to productive coughs, fever, loss of appetite and night sweating of their child, which had gradually begun at the time of the axillary mass growth.

Although there was a negative history of previous hospital admission or any apparent serious illness, the patient had several visits to the pediatrician during the first two months of life due to the described nodule and the parents had been reassured many times about the nature of the nodule which was described by doctors as a reaction to BCG vaccine sometimes seen in newborns after vaccination.
Authors

Zahra Movahedi ${ }^{1}$

Sayna Norouzi ${ }^{2}$

Setareh Mamishi ${ }^{3}$

Nima Rezaei ${ }^{4}$

${ }^{1}$ Fellowship of Pediatric Infectious Diseases, Pediatric Infectious Diseases Research Center, Tehran University of Medical Sciences, Tehran, Iran

${ }^{2}$ Intern, Division of

Pediatric Infectious

Diseases, Children's

Medical Center, Tehran

University of Medical

Sciences, Tehran, Iran

${ }^{3}$ Professor, Head of

Research Center and

Division of Pediatric

Infectious Diseases,

Pediatric Infectious

Diseases Research Center,

Tehran University of

Medical Sciences, Tehran,

Iran

${ }^{4}$ Assistant Professor of

Clinical Immunology

and Executive Director of

Children's Medical Center,

Molecular Immunology

Research Center; and

Research Center for

Immunodeficiencies;

Department of

Immunology, School

of Medicine, Tehran

University of Medical

Sciences, Tehran, Iran

Submitted on: 07/01/2010 Approved on: 07/31/2010

Correspondence to:

Setareh Mamishi

Pediatric Infectious

Diseases Research Center,

Children's Medical Center

Dr. Gharib St, Keshavarz

Blvd

Tehran 14194, Iran

Phone/Fax:

+982166428996

smamishi@tums.ac.ir

We declare no conflict of interest. 
At the time of admission, the patient was ill, but not toxic; she had low grade fever. At pulmonary auscultation of the upper and middle lobe of the right lung breathing sounds were increased and coarse. A firm illdefined tender mass was palpated in the right axillary region, which was extended to the right anterior chest wall about the midclavicular line of the third intercostals space (Figure 1 ).

Figure 1: Scar of the lesion in the right axillary region, extended to the right anterior chest wall.

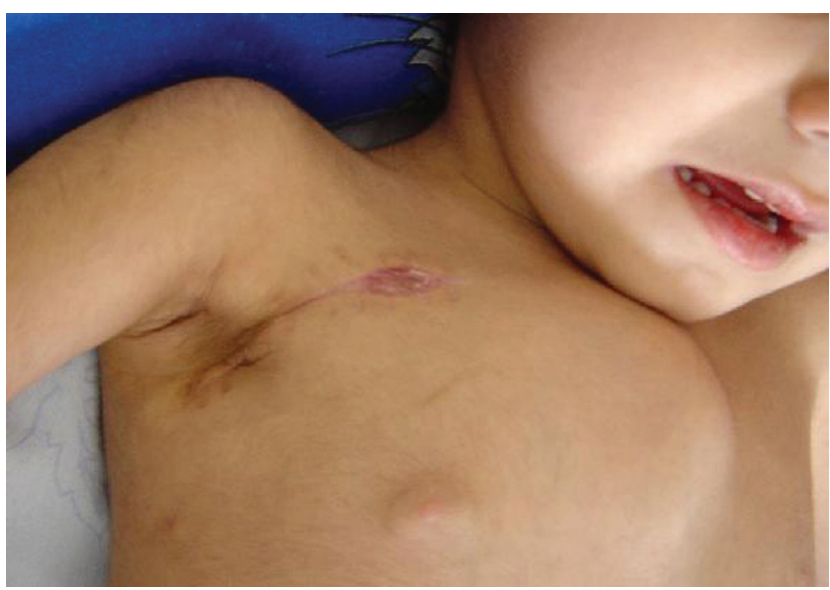

Laboratory tests and imaging studies were performed. The primary laboratory findings were as follows: white blood cell count: $17,800 / \mathrm{mm}^{3}$ (neutrophils: $69 \%$ and lymphocytes: 31\%), hemoglobin concentration: $10.7 \mathrm{~g} / \mathrm{dL}$, platelet count: $449,000 / \mathrm{mm}^{3}$, and erythrocyte sedimentation rate (ESR): $118 \mathrm{~mm} / \mathrm{h}$. The patient had a $4+$ positive C-reactive protein $(\mathrm{CRP})$. Chest roentgenogram (CXR) revealed a homogenous density with an alveolar-filling pattern in the right upper and middle lobes with a small lucency within the upper lobe density, which suggested a cavity forming pneumonia (Figure 2).

Considering the history of chronic cough, staining of sputum for acid-fast bacilli (AFB) was performed for the patient. Meanwhile, spiral computed tomography (CT) scanning of the chest with intravenous contrast enhancement was done for further evaluation of the chest wall mass and also for prompt diagnosis of findings on the CXR. The chest CT scan reported alveolar opacity with air bronchogram in right upper and middle lobes and also left lingual lobe suggestive of pneumonia and mediastinal lymphadenopathy (Figure 3), all together compatible with primary tubercu- losis infection. There was also a cystic mass (continuation of the axillary mass, which was $55 \times 24 \mathrm{~mm}$ in size) with rim enhancement anterior to the sternum that could be an abscess.

These findings raised the question about the competency of the immune system of the child. Hence, a series of the immune system assessment studies were done.

Figure 2: Chest x-ray of the patient showing a homogenous density with an alveolar-filling pattern in the right upper and middle lobes.

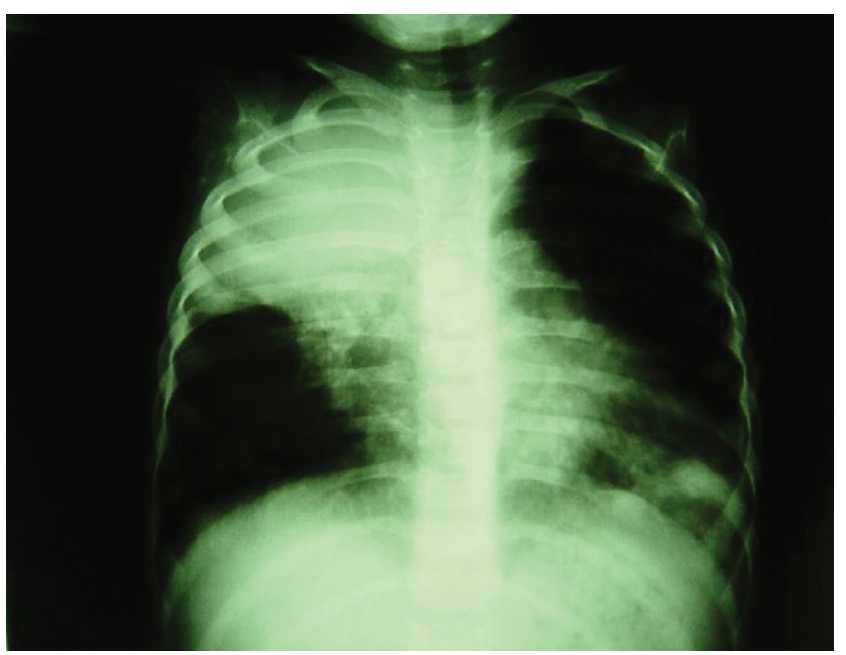

Figure 3: Chest CT scan of the patient showing alveolar opacity with air bronchogram in right upper and middle lobes.

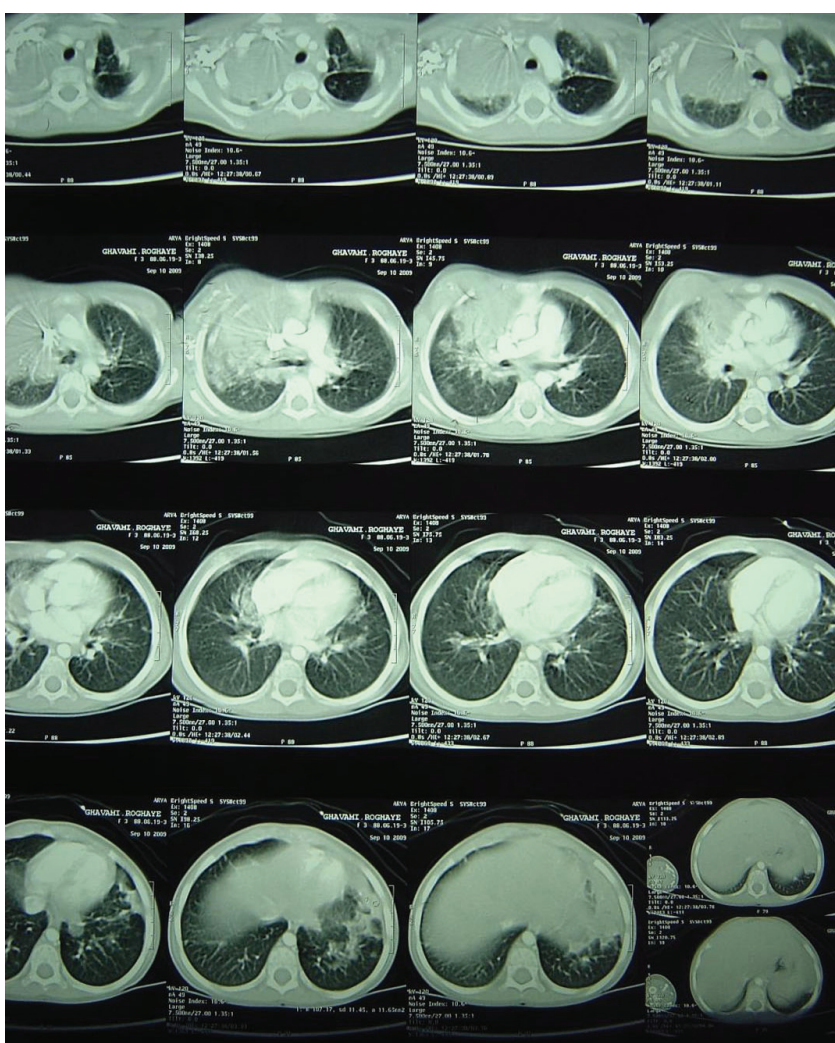


Serum IgG level was 2,136 mg/dL (normal: 441-1,135 $\mathrm{mg} / \mathrm{dL})$. Serum IgM was in high normal range $(332 \mathrm{mg} /$ $\mathrm{dL})$ and IgA was within normal range $(243 \mathrm{mg} / \mathrm{dL})$. HIV ELISA test was negative. However, nitroblue-tetrazolium (NBT) slide test, which is performed to assess the phagocytic cell function, revealed a defect in phagocytic cell function (NBT: 0\%), which was compatible with diagnosis of CGD.

The chest wall abscess underwent needle aspiration and sent for acid fast stain and routine $\mathrm{KOH}$ smear. Staining of the aspirated fluid disclosed Aspergillus fumigates. Besides, all three specimens of gastric lavage turned out positive for AFB; therefore conventional anti-TB regimen and anti-fungal therapies were administered for the patient. The abscess was once again aspirated and got smaller.

As the diagnosis of CGD was made, the patient received interferon-gamma (IFN- $\gamma 50 \mu \mathrm{g} / \mathrm{sc} / 3$ times in week), streptomycin, isoniazid, ethambutol, rifampin, and amphotericin $\mathrm{B}$.

Two weeks after anti-TB and anti-fungal therapy, the child's appetite had improved and the respiratory complaints declined. The mass size became smaller and the patient was discharged in good clinical condition.

\section{DISCUSSION}

In this case study, an atypical feature of CGD, a child primarily presented with BCG-induced lymphadenitis which progressed to BCGosis after about three years. CGD is a rare but well-known congenital disorder of the immune system, with an estimated 10-year cumulative incidence rate of 2.25 per 100,000 individuals in our country. ${ }^{9}$

The majority of CGD patients suffer from recurrent infection caused by Staphylococcus species, Gram-negative bacilli (like Escherichia coli or Klebsiella), and also saprophytic fungi, particularly Aspergillus species. ${ }^{5-7}$ Here, the patient presented with a rare but important complication of BCG vaccine, lymphadenitis. Overall, BCG vaccine is safe, but it may lead to a number of complications such as localized ulceration regional lymphadenopathies, which occur after inoculation and is managed by just waiting and watching unless it becomes fluctuated or shows an overgrowth in size. ${ }^{10}$ It is believed that some primary immunodeficiency diseases, including severe combined immunodeficiency, CGD and Mendelian susceptibility to mycobacterial diseases, are prone to severe mycobacterial disease following BCG vaccination. ${ }^{11-13}$

Surely, one of the most important aspects in optimal management of chronic diseases, like CGD, is the time of diagnosis. Early diagnosis would lead to a more aggressive management at the initial stages of the disease which could in turn, decrease complications. In Iran, the diagnostic test for CGD is based on the results of either
NBT or dihydrorhodamine (DHR) 123 test. The response of NBT test depends on the competence of normal neutrophils to reduce the colorless NBT dye to blue formazen during phagocytosis. Conclusively, as a result of the absence of minimal oxidative metabolism in CGD, a negative test is diagnostic. Although this exact diagnosis is achieved by the test mentioned above, a strong clinical suspicion is essential. Beyond any doubt, in such an atypical case, as presented here, understanding rare clinical manifestations could prompt early request of diagnostic tests like NBT. It is worthy to declare that although $\mathrm{x}$-linked CGD is the most common types of disease (about 60\%) in the western countries, autosomal recessive form of the disease seems to be more common in Middle East countries due to high rate of consanguineous marriages. ${ }^{14}$ Autosomal recessive inheritance was also expected in our case, as the patient was a girl.

Other interesting features of this case which indeed had an atypical presentation, is the patient's age at the time of disease presentation. CGD is a hereditary disease, characterized by severe infections of various body organs mostly present in the first year of life. ${ }^{6,7}$ In this case the only disease manifestation was an axillary nodule which remained unchanged during a 3-year period. Hence, this case could be considered as a late-onset form of the disease.

Finally, it seems that the clinical significance of this case report is the suggestion of persistent axillary lymphadenitis as a sentinel sign for immune disorders, particularly CGD. Surely, prompt diagnosis of such diseases would lead to a better clinical outcome, as for this case. Early administration of gamma interferon and itraconazole prophylaxis, for example, might prevent disseminated BCGosis and secondary fungal infection of the inflamed lymph node. Since the routine national vaccination program in Iran includes BCG vaccine, CGD as well as other primary immunodeficiency diseases, such as severe combined immunodeficiency and Mendelian susceptibility to mycobacterial diseases should be considered in the patients with disseminated disease after BCG inoculation.

\section{REFERENCES}

1. Winkelstein JA, Marino MC, Johnston RB Jr et al. Chronic granulomatous disease. Report on a national registry of 368 patients. Medicine (Baltimore) 2000; 79:155-69.

2. Martín Mateos MA, Alvaro M, Giner MT et al. Chronic granulomatous disease: six new cases. Allergol Immunopathol. (Madr) 1998; 26:241-9.

3. Movahedi M, Aghamohammadi A, Rezaei N et al. Chronic granulomatous disease: a clinical survey of 41 patients from the Iranian primary immunodeficiency registry. Int Arch Allergy Immunol. 2004; 134:253-9.

4. van den Berg JM, van Koppen E, Ahlin A et al. Chronic granulomatous disease: the European experience. PLoS One 2009; 4:e5234. 
5. Cohen MS, Isturiz RE, Malech HL et al. Fungal infection in chronic granulomatous disease. The importance of the phagocyte in defense against fungi. Am J Med. 1981; 71:5966.

6. Johnston RB Jr. Clinical aspects of chronic granulomatous disease. Curr Opin Hematol. 2001; 8:17-22.

7. Martire B, Rondelli R, Soresina A et al. IPINET. Clinical features, long-term follow-up and outcome of a large cohort of patients with Chronic Granulomatous Disease: an Italian multicenter study. Clin Immunol. 2008; 126:155-64.

8. Rosenzweig SD. Chronic granulomatous disease: complications and management. Expert Rev Clin Immunol. 2009; 5:45-53.

9. Rezaei N, Aghamohammadi A, Moin M et al. Frequency and clinical manifestations of patients with primary immunodeficiency disorders in Iran: update from the Iranian Primary Immunodeficiency Registry. J Clin Immunol. 2006; 26:519-32.
10. Grange JM. Complications of bacille Calmette-Guérin (BCG) vaccination and immunotherapy and their management. Commun Dis Public Health 1998; 1:84-8.

11. Rezai MS, Khotaei G, Mamishi S et al. Disseminated Bacillus Calmette-Guerin infection after BCG vaccination. J Trop Pediatr. 2008; 54:413-6.

12. Sadeghi-Shabestari M, Rezaei N. Disseminated bacille Calmette-Guérin in Iranian children with severe combined immunodeficiency. Int J Infect Dis. 2009; 13:e420-3.

13. Reichenbach J, Rosenzweig S, Doffinger R et al. Mycobacterial diseases in primary immunodeficiencies. Curr Opin Allergy Clin Immunol. 2001; 1:503-11.

14. Rezaei N, Pourpak Z, Aghamohammadi A et al. Consanguinity in primary immunodeficiency disorders; the report from Iranian Primary Immunodeficiency Registry. Am J Reprod Immunol. 2006; 56:145-51. 